\title{
Multilinguales
}

15 | 2021

Recherches algériennes en sociolinguistique et en littérature : discours scientifique/discours militant, quelles frontières?

\section{Deux romans de Kamel Daoud, entre militantisme satirique et symbiose interculturelle}

Two novels by Kamel Daoud, between satirical activism and intercultural symbiosis

\section{Smail Mahfouf}

\section{(2) OpenEdition}

\section{Journals}

Édition électronique

URL : https://journals.openedition.org/multilinguales/6446

DOI : $10.4000 /$ multilinguales.6446

ISSN : 2335-1853

Éditeur

Université Abderrahmane Mira - Bejaia

Référence électronique

Smail Mahfouf,

«Deux romans de Kamel Daoud, entre militantisme satirique et symbiose interculturelle»,

Multilinguales [En ligne], 15 | 2021, mis en ligne le 15 juin 2021, consulté le 14 février 2022. URL : http:// journals.openedition.org/multilinguales/6446; DOI : https://doi.org/10.4000/multilinguales.6446

Ce document a été généré automatiquement le 14 février 2022.

\section{(c) (i) (5)}

Multilinguales est mise à disposition selon les termes de la Licence Creative Commons Attribution Pas d'Utilisation Commerciale - Pas de Modification 4.0 International 


\section{Deux romans de Kamel Daoud, entre militantisme satirique et symbiose interculturelle}

Two novels by Kamel Daoud, between satirical activism and intercultural symbiosis

Smail Mahfouf

1 Notre choix du corpusZabor ou les Psaumes(2017) etLe peintre dévorant la femme

(2018) est motivé par la thématique du sacré qui en constitue la toile de fond. De ce sacré à l'œuvre découle notre démarche analytique. Nous considérons, en effet, qu'à

l'arrière-plan de ces romans se profile une forme de dialogue interculturel, qui implique dans une large mesure le religieux. Cette dynamique interculturelle, qui est

Meursault, contre-enquête

amorcée et limitée dans le premier roman,

(2013) à l'histoire coloniale franco-algérienne se trouve étendue dans

Zabor ou Les PsaumesetLe peintre dévorant la femme

aux imaginaires oriental et occidental grâce à une forte inscription du mythe, de l'art et

du religieux. Ce dernier étant essentiellement constitué de récits bibliques, de versets

coraniques et de hadiths 1. 
Plus qu'un contenu commun aux deux romans, ce sacré détermine la méthodologie même à mener, à savoir que cette thématique est à l'origine du caractère ambigu de l'écriture romanesque de Kamel Daoud. En effet, à ses balbutiements, cette écriture se fait expression politique, un militantisme dirigé contre le religieux jugé être derrière l'anachronisme culturel, particulièrement dans le monde arabe. Cet énoncé politique

du projet romanesque, apparent, de Kamel Daoud est assumé par l'écrivain, grâce notamment à ses intrusions fréquentes, qui relèguent au second plan la fiction narrative. En même temps, ce projet créateur se révèle être une contre-expression du militantisme entrepris, c'est-à-dire que la spiritualité, qui est par ailleurs érigée en une cible privilégiée du discours satirique fortement à l'œuvre, devient vecteur d'une symbiose interculturelle pluridimensionnelle. Ce devenir interculturel du sacré s'accomplit grâce au dialogue que cette spiritualité entretient avec les composantes essentielles de l'art, du mythe et de l'écriture.

L'analyse de cette ambiguïté romanesque qui spécifie l'écriture des deux romans se fera en deux parties successives et complémentaires. La première concerne l'énoncé politique de cette écriture, ce discours idéologique impliquant la moralité de l'écrivain et sa visée de la refonte culturelle dans le monde arabe. D'où la formule « Le sacré, cible d'un rabaissement satirique » qui résume cette dimension politique de l'écriture daoudienne. La seconde partie de l'analyse consistera à démontrer que ce même sacré s'offre comme une esthétique interculturelle en mouvement. C'est cette transmutation

interculturelle du sacré que résume la formule : « Le sacré, facteur d'une symbiose interculturelle ». Dans ce qui suit, nous allons d'abord examiner l'allure discursive de cette écriture puis ses contours esthétiques.

\section{Le sacré, cible d'un rabaissement satirique}

Le volet politique de l'écriture de Kamel Daoud concorde avec la présence massivedes références religieuses dans

Zabor ou les PsaumesetLe peintre dévorant la femme

. Ce discours s'en tient à ces références religieuses en les rabaissant. Il se trouve, de ce fait, en grande partie soustrait au voile de la fiction, si bien que l'instance narratrice cède devant les implications manifestes de l'écrivain-orateur. En rhétorique, cette stratégie discursive est désignée par le concept de

l'éthos,

c'est-à-dire : « Les traits que l'orateur doit montrer à l'auditoire pour faire bonne impression ; ce sont ses airs » (Barthes, 1985 : 146). Kamel Daoud agit en orateur dans son œuvre, il exerce une influence persuasive sur ses lecteurs, en multipliant dans ce sens ses attaques à l'encontre de l'héritage spirituel en présence. Dans

Zabor ou les PsaumesetLe peintre dévorant la femme

la satire prend sens de cette attitude extrêmement critique. 
Il convient, pour mieux vérifier l'emploi de la satire dans les deux romans de Kamel Daoud, d'interroger cette notion, d'en préciser l'étymologie. Le mot satire est latin, « Satira",

selon Le Petit Robert de la langue française, ce mot renvoie à « Un ouvrage libre de la littérature latine où les genres, les formes, les matières étaient mêlées et qui censurait les mœurs publiques » (Le petit Robert de la langue française, 2015 : 2312). La satire cible une norme axiologique, c'est-à-dire (tout système de valeurs religieuses, politiques, morales ou sociales). La satire se définit également par sa finalité pragmatique, en d'autres termes, la stratégie rhétorique, dont elle se sert, vise à rabaisser la valeur sociale, ciblée, dans l'esprit du public.

DansZabor ou Les psaumesetLe peintre dévorant la femme

de Kamel Daoud, c'est sur la norme axiologique du sacré que porte l'expression satirique de rabaissement. Et cette présence du sacré est signalée dès l'ouverture des deux romans,

Zabor ou les Psaumes

commençant par un verset coranique « Noun ! Et le calame et ce qu'ils écrivent »

(Daoud, $2017: 13)$;

Le peintre dévorant la femme

inscrit l'élément religieux de « la pierre noire » qui se trouve à la Mecque « Paris est une pierre sacrée blanche » (Daoud, 2018 : 9). Les deux récits multiplient au fur et à mesure de leurs évolutions ces références religieuses en les soumettant à deux principales formes d'expressions satiriques complémentaires, celle d'une rhétorique offensante, et celle d'une parodie à la tonalité fortement ironique. Dans ce qui suit, nous examinerons deux formes principales de cette rhétorique injurieuse, à savoir le sarcasme et le mépris, ce dernier souvent introduit par des allégories.

\subsection{Une rhétorique offensante}

La rhétorique injurieuse est la principale forme d'expression satirique, militante, chez Kamel Daoud. Elle est essentiellement sarcastique, et le mot sarcasme étant dit en grec, son sens étymologique,

sarcasmus

en latin, qui signifie, selon le Petit Robert de la langue française (2015), « Mordre la chair ». Appuyée sur cette étymologie, l'analyse du ton sarcastique dans l'œuvre de Kamel Daoud s'associe à un autre concept de la rhétorique, celui de

l'éthos

en ses deux aspects de moralité, ou de vérité que l'auteur prétend détenir, et de mépris soutenu par un puissant esprit critique. Le premier met en avant le profil moderniste de Kamel Daoud, ses idées progressistes par lesquelles il exerce davantage d'influence sur ses lecteurs, à les faire adhérer à sa vision de la culture. C'est pourquoi ce discours intellectualiste est entaché d'un certain moralisme que trahissent les formules : «Je m'égare » (Daoud, $2017: 22)$ ), « Je me perds » (Daoud, $2017: 17)$, « J'en suis sûr »

(Daoud, $2018:$ p. 83), «J'en ai honte » (Daoud, $2017: 217$ ). 
L'investissement de l'éthos

moral de l'écrivain est lié au paradigme de la création opposé à celui du sacré tout le temps vilipendé. Cette création est évoquée par le biais des thèmes sensibles d'une écriture libre de toute censure morale, de l'art du corps, du cannibalisme, de l'érotisme, autant de thèmes qui sont mis au service du projet politique, daoudien, de la refonte culturelle. La visée est toujours celle de chercher des stratégies de mobilisation de

l'auditoire, de stimuler son pathos,

c'est-à-dire "Les affects de celui qui écoute, tels que du moins l'orateur les imagine "

(Barthes, 1985 : 146). À cet effet, l'entremêlement de l'érotisme et de la culture constitue l'une de ces stratégies discursives mobilisatrices. En effet, la citation suivante exprime, selon le point de vue de Kamel Daoud, l'impact que pourrait avoir l'érotisme sur la culture, et l'écrivain se fait encore plus explicite en assimilant cet érotisme à sa vision du monde :

L'érotisme est une clef dans ma vision du monde, et de ma culture. Les religions sont l'autodafé des corps et j'aime, dans ce mouvement obscur de la dévoration érotique, la preuve absolue que l'on peut se passer des cieux, des livres et des temples. L'érotisme est la permanence de l'homme, la preuve que l'au-delà est un corps que l'on a sous la main et dans le ventre ici et pas "après" (Daoud, $2018: 16$ ).

La jonction établie entre la personne de l'énonciateur et l'érotisme est fortement marquée. Cette manœuvre procède d'un parti-pris idéologique, à savoir une plaidoirie en faveur d'une émancipation culturelle en totale rupture avec l'éthique religieuse.

L'érotisme, comme issue possible à l'enfermement culturel, se fait aussi stratégie carnavalesque, dont parle Mikhaïl Bakhtine, c'est-à-dire l'association du culturel au bas matériel, au physiologique, au scatologique, au sexuel.

\section{L'autreéthos}

qui participe à la structuration de l'expression sarcastique, satirique, dans l'œuvre de Kamel Daoud est celui du mépris. Dans cette autre forme de satire, le référentiel prime toujours sur le fictionnel, par conséquent la norme axiologique, religieuse, demeure la cible du discours méprisant à l'œuvre. Doublement représentée, cette norme axiologique revêt deux formes principales : l'une, explicite par l'évocation directe de la spiritualité et l'autre, implicite présentée sous la forme d'allégories. Les entités de Dieu, du destin, du Livre sacré, de la prière et du paradis composent cette dimension explicite du sacré. Le propos, tenu à l'égard de ces valeurs religieuses, est

fréquent et ouvertement moqueur. Quatre exemples de propos, que nous allons consécutivement citer, convergent vers un même sens, celui des formules : «Dans le Livre sacré l'histoire des frères jaloux finit bien pour la victime, mais dans la vie c'est différent. Dieu manque parfois d'inspiration » (Daoud, $2017: 41)$. « Le destin est un cahier comportant des fautes que l'on peut corriger » (Daoud, $2017: 112$ ). « Le jugement n'est pas dernier, il est permanent » (Daoud, $2018: 10)$. « Le paradis fait partie de la vie pas de la mort !» (Daoud, $2018: 30$ ). Et le dernier exemple est : « Quand le livre est sacré l'homme ne l'est plus » (Daoud, $2018: 32$ ). Ces formules de mépris, qui sont émises

à l'encontre du religieux, se caractérisent par un style lapidaire. En effet, la forme en slogan de ces énoncés favorise la mobilisation attendue. Par ailleurs, ce mépris se révèle sous une autre forme, celle de l'allégorisation du sacré qui intègre de nombreux et divers éléments matériels de la nature. 
L'allégorisation du sacré s'illustre dans les figures matérielles du Sahara, du vent, de la pierre tombale ou du cimetière, du mouton, du couscous, une diversité d'éléments hétérogènes qui atteste la place centrale qu'occupe cette thématique dans l'œuvre de Kamel Daoud. Dans les passages suivants cette matérialité est à la foisgéographique, atmosphérique, zoologique, pharmaceutique et culinaire : « L'odeur du couscous qui est une odeur de mort s'infiltre et s'ajoute à celle de l'acide agonie, des médicaments et des

élevages rances des moutons » (Daoud, $2017: 29$ ). «L'odeur du couscous grasse et lourde, on le prépare toujours en même temps que le cercueil et la noce » (Daoud, 2017 : 47). « Les cimetières c'est de la friperie. Un débarras d'habits. De l'éternité mal cousue »

(Daoud, 2017 : 48). « Le Sahara avait quatre-vingt-dix-neuf noms lui aussi, et lui aussi était invisible et colérique » (Daoud, $2017:$ p. 68). «Je me suis mis à penser aux vents que j'ai toujours détestés (le Prophète demande à ce qu'on n'insulte pas le vent, car c'est un signe de l'esprit » (Daoud, $2017: 43$ ).

Ce qui n'est pas explicitement dit dans les premières catégories sacrales l'est dans ces allégories, à savoir l'identification du sacré à la mort. Dans le projet de Kamel Daoud, cette mort est essentiellement culturelle, et l'orateur en fait un argument mobilisateur. Du renforcement de son éthos

moral, en vue de convaincre et de mobiliser, au mépris exprimé, Kamel Daoud pousse à son paroxysme le discours satirique à l'encontre du sacré. Ce discours se prolonge dans une autre forme satirique, celle d'un jeu intertextuel.

\subsection{Les retournements intertextuels}

La stratégie intertextuelle adoptée dans l'œuvre de Kamel Daoud est de forme subtile, atténuée. Les deux romans acquièrent le statut d'hypertexte où se poursuit la dégradation du corpus coranique, biblique, en tant qu'hypotexte. Dans ce contexte, Julia Krestiva, se référant au principe dialogique de Mikhail Bakhtine, explique ce

phénomène de transformation intertextuelle. Ainsi : « Tout texte se construit comme une mosaïque de citations, tout texte est absorption et transformation d'un autre

Zabor ou Les PsaumesetLe peintre dévorant la femme

transforment triplement le monothéisme religieux : par la citation aux accents ironiques, par la transposition thématique et par le travestissement burlesque. La citation, forme emblématique de l'intertextualité, se manifeste par « La présence effective d'un texte dans un autre, sous sa forme la plus explicite et la plus littérale (avec guillemets avec ou sans référence précise » (Genette, 1982 : 8). Si par son évidence, cette inscription n'exige pas une quelconque perspicacité du lecteur, elle est, néanmoins, de nature à inciter à s'interroger sur la fonction qu'elle est censée remplir dans le texte. 
Dans les deux romans précités de Kamel Daoud, qui sont soumis à l'étude, la fonction assumée par les nombreuses citations religieuses est celle de l'ironie, les citations imbriquées dans les deux récits est déjà une manière de tourner leur contenu spirituel en dérision. Vladimir Jankélévitch précise cet usage de la citation en parlant d' « Une circonvolution du sérieux » (Jankélévitch, 1964 : p. 58), ou un sérieux tu. Cette évacuation du sérieux est, par exemple, illustrée dans les versets coraniques suivants : "Tandis que les poètes sont suivis par les égarés / Ne les vois-tu pas errer dans chaque vallée.../... et disent ce qu'ils ne font pas?» (Daoud, $2017: 24)$. « Donnez vie à vos images si vous en êtes capables!» (Daoud, $2018: 159$ ). La reprise ironique - par Kamel Daoud - de ces versets consiste à sous-entendre leurs sens contraires, latents, celui de l'encensement de l'égarement et de l'errance des poètes dans le premier verset et celui d'une création artistique libre, aussi scandaleuse soit-elle, dans le second.

L'autre procédé de l'altération du sacré est celui de la transposition thématique- celleci étant d'une grande amplitude textuelle, car pouvant s'étendre à tout un texte -qui touche la signification même de l'hypotexte. Selon Gérard Genette, cette transposition relève de deux ordres : le premier ordre est diégétique, c'est-à-dire spatio-temporelle, alors que le deuxième ordre est pragmatique (

\section{de pragmma}

qui signifie, selon le petit Robert de la langue française, (2015) « Evénement, action », dans ce dernier cas, l'hypertexte modifie l'intrigue ou le cours des événements, de l'action. Dans les récits de Kamel Daoud, ce déplacement de l'espace, du temps et de l'action concerne, à titre d'exemple,l'histoire du Prophète Moïse, qui se trouve transposée dans l'époque contemporaine du terrorisme international. Le propos qui suit illustre ce cas de figure :

J'imagine donc un autre personnage qui serait né en Occident, ou pas, enfant sans père ni mère, né du zénith et de la nuit, ou enfant presque illégitime confié aux courants d'eau, comme Moïse, mais cette fois noyant son peuple. [...] Traduire : un enfant de l'histoire immédiate ou un orphelin qui rêve de détruire les généalogies du monde pour se sentir réparé (Daoud, 2018 : 51).

L'intégration de ce récit médiéval dans l'actualité terroriste et la substitution de l'acte prophétique de sauvetage - Moïse franchissant la mer par son bâton - par celui profane de la noyade se double d'une autre défiguration avilissant encore davantage le religieux, celle de la réincarnation de Moïse dans le personnage « illégitime » cité. Ainsi, l'amplitude de ces catégories narratives est à la mesure de celle de la transformation introduite dans ce récit prophétique.

Enfin, le travestissement burlesque sert également de biais à l'expression satirique de la dégradation du religieux chez Kamel Daoud. Ce travestissement s'opère par une transformation stylistique du texte noble en maintenant intact le sujet de celui-ci. Ce cas de figure se présente, entre autres, dans la réécriture d'une autre histoire sacrée du

Zabor ou Les Psaumes.

Le rabaissement stylistique réalisé est relatif au sujet noble du sacrifice. En effet, la citation suivante est une caricature de l'événement sacré en question :

Le mouton avait levé les yeux d'une douceur poignante, avait interrompu son éternité et s'était éparpillé en mille bêtes destinées à détourner le regard de Hadj Brahim de ma personne. Pour m'épargner, il s'était donné au patriarche, et m'avait offert la puissance de l'écrivain capable de contrer la mort (Daoud, 2017 : 83). 
En effet, le mouton céleste venu sauver le fils d'Abraham est resté sans modification au niveau de l'hypertexte. Cependant, le style, dans lequel est relatée cette histoire, est tout autre que celui du Coran ou de la Bible. Cette trivialisation stylistique du religieux consiste à personnifier le mouton, ce dernier compatit avec le triste sort d'Ismaël et il octroie au narrateurle don de l'écriturecréatrice.

L'œuvre de Kamel Daoud se fait - du moins à ce stade - discours satirique en vue de rabaisser le sacré. L'omniprésence de la norme axiologique, religieuse, plaide en faveur de cette hypothèse de l'essence polémique de l'écriture de Kamel Daoud. Cependant,

limiter cette œuvre romanesque à un simple discours militant, c'est faire passer promptement sous silence son expression fictionnelle, implicite, brefévacuer injustement la force créatrice de cette écriture. Saisir donc cette œuvre dans sa complexité, implique de s'attarder sur sa poétique secrète, de tenter d'appréhender le devenir fictionneldu sacré. À ce titre, la nouvelle question qui se pose est celle : Qu'en est-il de la poétique du sacré comme contre-expression du projet militant précédent ? Cette poétique est un processus textuel où le sacré devient une symbiose interculturelle.

\section{Le sacré, facteur d'une symbiose interculturelle}

Souvent présenté sous la forme de biographies de prophètes, le sacré se trouve associé au mythedu salut, présenté comme une exaltation d'actes de délivrance. Ce récit fondateur de la foi religieuse correspond à ce que le mythologue roumain, Mercia Eliade, appelle le mythe de l'origine qu'il définit en ces termes : « Une histoire sacrée, un événement qui a eu lieu dans le temps primordial, le temps fabuleux des commencements » (Eliade, 1963 : 15). Dans les deux romans de Kamel Daoud, le temps primordial est celui des Prophètes, et les récits sont tous ceux du salut.

Dans les religions monothéistes, ces histoires sont nombreuses : histoire du prophète joseph tiré du puits où l'ont mis ses frères jaloux « Le loup l'a mangé, ont dit les frères au patriarche aveugle et éploré » (Daoud, $2017: 290$ ) ; histoire du mouton céleste grâce auquel fut sauvé le Prophète Ismaël, fils d'Abraham ; histoire du Prophète Nouh, ou Noé, sauvé lui et ses compagnons du déluge ; histoire du Prophète Jonas, ou Younes, allant à la rencontre de Dieu « Qui s'est manifesté à lui sous la forme d'un navire

ancien, d'une tempête haineuse, de marins, puis d'une baleine, puis d'un arbre qui donne son ombre au corps nu du naufragé » (Daoud, $2017: 248$ ), etc. Tissé sur la toile de fond du mythe salvateur élargissant le dialogue entre contes, arts et écritures, le projet

Zabor ou Les PsaumesetLe peintre dévorant la femme

recrée ce substrat sacral du salut en une symbiose interculturelle. Les contes

Mille et Une NuitsetRobinson Crusoé,

qui constituent la première section du deuxième axe de notre article, participent à cette dynamique interculturelle. C'est cette autre dimension dialogique qui sera examinée. 


\subsection{Robinson Crusoé et Mille et Une Nuits}

La relation dialogique entre lesMille et Une NuitsetRobinson Crusoé

de Daniel Defoe paraît dans le triomphe de l'imagination créatrice sur la mort qui spécifie ces deux contes. Les

Mille et Une Nuits

mettent en scène un antagonisme entre deux forces, celle d'une parole conteuse s'ingéniant à inventer toujours mieux, pour mieux « Justifier la méfiance du roi cocufié

[Shahrayar] envers les femmes " (Bencheikh, $1988: 29)$ et celle d'une exécution menaçant de s'effectuer au moindre tarissement de la parole en exercice. Shahrazade vainc la mort, elle humanise le roi et devient, au terme de cette périlleuse aventure, son épouse. Elle se sauve donc, sauve d'autres femmes, et c'est, en définitive, en un modèle féminin sacré aux pouvoirs créatifs, illimités, que cette héroïne s'érige dans

l'imaginaire oriental.

Robinson Crusoé

est l'histoire d'un naufragé, un Robinson se trouvant sur une île sans trace humaine ni repère culturel, sauf la bible lui servant à la fois de consolation et de source d'improvisation de quelque forme de vie sur l'île. À l'instar de Shahrazade, Robinson doit vaincre la mort, mettre à rude épreuve son imagination à même d'apprivoiser cet univers sauvage et inhospitalier de l'île, d'y faire naître la vie.

De ces contes, où la vie mime constamment la mort, Kamel Daoud en fait une métaphore interculturelle. En effet, l'enjeu dépasse les deux contes (dans l'un, sauver une femme face à un roi féminicide et un homme perdu sur l'île dans l'autre), il s'agit de sauver la culture, lui trouver d'autres attaches plus flexibles avec le religieux; d'où le biais du conte qui n'est, à ce titre, qu'une stratégie qui vise la réparation culturelle envisagée. Dans cette veine, toutes les citations du récit

Zabor ou Les Psaumes, associées auxMille et Une Nuits,

mettent en avant la culture arabesymbolisée parle village fictionnel d' " Aboukir »,

«Centre du monde situé entre mon nombril et mon cœur» (Daoud, $2017: 15$ ).

La langue arabe occupe aussi une place privilégiée dans ce projet culturel ; elle est décrite dans toute son inertie, tout à l'opposé de la parole vivifiante des

Mille et Une Nuits

. Dans le récit de Kamel Daoud, cette parole est assumée par le personnage de Hadjer, la tente du narrateur : «Elle repousse ta décapitation par ses Mille et Une Nuits improvisées, sa verve et sa ruse » (Daoud, 2017 : 144). Or si telle est la langue fertile du conte, la langue arabe, telle que pratiqué dans le village précité, « Il [lui] manquait le rêve, le mystère du conte comme je le comprenais plus tard. Nous étions un pays récemment libéré de la colonisation et les mots se faisaient soldats » (Daoud, 2017 : 144). La formule« les mots se faisaient soldats » résument le figement de la langue et de la culture arabes. Inspiré des

Mille et Une Nuits,

le besoin de créer une langue vivante est exprimé par une métaphore interculturelle, vive, celle d'un livre à écrire par une combinaison aléatoire de tous les autres. Cette réalisation inclut tout le sens du rêve et du mystère qui font la force du conte. La biographie du narrateur est quasi-identique à ce livre en devenir. En voici un fragment : 
Il y a quelques années après mon passage par l'école coranique, j'en ai accentué le désordre en décollant les couvertures de certains pour les coller sur d'autres, créant ainsi un brouhaha insonore, un amalgame qui augmente la combinaison possible des textes et de leur sens. Une sorte d'autodafé inédit, démiurge. Comme si je jetais les romans en l'air et qu'ils retombaient riches de nouvelles pistes, par le hasard de rencontres et de collisions entre titres vierges et récits orphelins (Daoud, 2017 :

196).

L'improvisation ingénieuse desMille et Une Nuits

devient la démarche créatrice par laquelle s'accomplirait le livre à venir, de son sens tout neuf issu d'autres livres se recombinant. En filigrane de ce procédé créateur, interculturel, se profile la démarche surréaliste de jeux collectifs, dit « cadavre exquis », ou d'écriture automatique qu'André Breton définit comme « Le fonctionnement réel de la pensée » (Breton, 1920 : 42) Cette méthode tiendrait lieu d'instrument de reconstruction interculturelle, à la manière du surréalisme et des Mille et Une Nuits.

ce projet interculturel, qu'incarne la mosaïque de livres en perpétuelle recomposition, contraste avec la phrase répétitive du perroquet Poll, celle « Pauvre Robinson où estu ? ». À la différence de la dynamique interculturelle de livres s'entremêlant, cettephrase statiqueserait synonyme d'une stagnation culturelle. Dans la citation suivante, un lien est établi entre cette phrase de Poll et les textes sacrés qui sont également immuables et répétitifs :

Peut-être que nos langues n'avaient aux yeux du dieu déserteur que le sens d'une seule phrase réitérée sans cesse depuis des millénaires, recomposée à l'infini ? Peutêtre que le village où je vivais n'était qu'une île renfermée et sourde que j'étais chargé de libérer par de longs récits et l'apprentissage d'une langue plus vaste, plus vigoureuse, plus proche du naufragé que de ses perroquets qui tournaient en rond.

(Daoud, 2017 : 156).

À l'enfermement culturel s'oppose l'urgence interculturelle de faire « L'apprentissage d'une langue plus vigoureuse ", d'écrire-libérer de longs récits, comme projet interculturel salvateur qui fait écho au mystère et au rêve propre à la parole conteuse, toute aussi salvatrice des

\section{Mille et Une Nuits}

. Cette langue essentiellement créatrice est celle, par exemple, qui mêle dans les deux romans de Kamel Daoud les écritures romanesque et ésotérique. C'est ce que nous allons analyser dans la seconde section du deuxième axe 


\subsection{Ecriture romanesque et écriture ésotérique}

L'hybridité de l'écriture de Kamel Daoud est l'aboutissement d'une forme interculturelle particulière, qui est générée par la fiction du salut. L'intrigue de Zabor ou Les Psaumes

s'offre elle-même en exemple de ce type de fiction. En effet, de bout en bout du récit, l'acte de narration se confond avecl'acte de sauvetage salvateur, et le narrateur s'adossant tour à tour la mission de l'écriture pour faire reculer la mort dans son village : «Quand j'utilise les bons mots, la mort redevient aveugle et tourne en rond » (Daoud, 2017 : 19). Mais pour être pleinement salutaire, cette écriture doit se déployer en dehors, voire à contrecourant du Livre sacré. À cet égard, le propos du narrateur est

sans équivoque : «Je pouvais sauver une vie et congédier la mort en écrivant autre chose que leurs versets et les quatre-vingt-dix-neuf noms de Dieu » (Daoud, 2017 : 31). Improviser des formes d'écritures à l'écart du cadre légitimiste du Livre sacré aboutit à une prolifération d'écrits libres grâce auxquels bien des vies furent arrachées à la mort, dont celle de Hadj Brahim, le père du narrateur, le plus longuement assisté par une telle thérapie scripturaire.

La fusion des écritures romanesque et ésotérique, qui contribue au renforcement du processus interculturel dans les deux romans de Kamel Daoud, s'effectue sur le fond de

l'autobiographique. En effet, le narrateur évoque son enfance et son expérience de

l'apprentissage coranique. Le narrateur-adulte qui raconte cette phase sensible de sa vie y demeure encore fortement attaché, ce qui serait une manière à lui de revendiquer

cette Tradition. La citation suivante illustre clairement cet attachement :

J'adorais humer l'odeur de l'argile qui nous servait à enduire le bois pour le blanchir et accueillir les nouveaux versets. [...]L'odeur moisie du sansal, l'argile, sont des laines brûlées sont restées pour moi comme le parfum d'un mystère. Et si

le Livre sacré était parfois ennuyeux pour l'enfant que j'étais, avec des mots impossibles à comprendre, des hurlements et des récits trop épars pour accrocher

l'imagination, j'aimais méditer longuement sur les premiers mystères. (Daoud, $2017: 223)$.

Comme l'atteste clairement la dernière formule " comme l'écriture sous mes yeux cela devrait avoir un sens ", l'ésotérisme est la matrice sur laquelle s'élaborent les contours de l'écriture romanesque. La perméabilité du romanesque, sa disposition à intégrer d'autres genres en les remodelant, c'est la définition même qu'en donne Marthe Robert, lorsqu'elle souligne la capacité du genre romanesque à « S'emparer de secteurs de plus en plus vastes de l'expérience humaine » (Robert, 1972 : 15). Dans le cas de Zabor ou Les Psaumes,

cette expérience humaine est celle de l'expérience enfantine aux fortes prises avec

l'ésotérisme que le romanesque s'associe. En réinventant l'ésotérisme par le romanesque, Kamel Daoud élargit son esthétique interculturelle, cellecicommereconfiguration permanente du mythe du salut.

Cette esthétique interculturelle salvatrice, qui fait figure d'un ésotérisme romancé, prend l'allure d'undésordre scripturaire. Celui-ci est à l'image du mystère qui fonde la syntaxe ésotérique. Le passage suivant met l'accent sur le caractère irréductiblement 
Noun! Et le calame et ce qu'ils écrivent, dit le Livre sacré. Le roseau taillé comme un stylet, plongé dans l'encre du plus vieil océan. [...] Je voulais juste déplier ce livre d'une seule page [...] Et tout ce que je pus y lire, ce fut une écriture folle, désordonnée, dense comme une toison secrète, emmêlé de signes, de chiffres et d'exclamations, ponctuée d'astres et d'étoiles sommaires, incompréhensible comme un ciel constellé (Daoud, $2017: 189$ ).

Ainsi décrit, l'ésotérisme favorise la création par le biais de laquelle se renouvelle le processus interculturel dans l'œuvre de Kamel Daoud. Cette écriture ésotérique ne se présente pas dans un ordre syntaxique fixe et rigoureusement codifié, mais comme une recombinaison de signes hétérogènes, qui en rendent insaisissable le sens. C'est cette opacité, à la fois lexicale et sémantique, qui est derrière la transposition de l'ésotérisme dans le genre romanesque. Enfin, nous parachevons ce processus textuel de la transmutation interculturelle du sacré, en analysant, à présent, deux autres formes artistiques, en symbiose, celles du nu pictural et de la calligraphie arabe. Ces dernières constituent la troisième section du deuxième axe de notre étude.

\subsection{Nu pictural et calligraphie}

Le nu de Picasso et la calligraphiearabefont de la représentation du corps, celui féminin

en particulier, une issue au figement culturel dans le monde arabe. À l'instar des

Mille et Une Nuitset de la «Robinsonnade " ${ }^{2}$,

ces arts se trouvent dans la même relation mimétique avec la mort, car faisant face au triomphe de l'Invisible, à la résistance d'un imaginaire collectif, hostile. De ce fait, ces arts s'inscrivent en droite « Des icônes de l'ancien ordre païen », dit en transposition de

l'arabe au français «

Timthal »,

(Daoud, 2018 : 159), et donc perçues comme une « Hérésie ou idolâtrie » (Daoud, 2018 :

159). Il en résulte que ces œuvres d'art sont à détruire, comme ce fut le cas de « La statue de Sétif de Francis de Saint-Vidal datant de 1898 [...], dynamitée en 1997 par les groupes islamistes » (Daoud, $2018:$ p. 156).

Une fois de plus, Kamel Daoud fait de cet art du nu une autre métaphore interculturelle en fusionnant deux corps féminins, celui sacré de Houri, «Cette femme promise après la mort » (Daoud, 2018 : 10) et celui profane de Marie Thérèse, " Une jeune femme de dix-huit ans que Picasso rencontre au hasard à Paris en janvier 1932 (Daoud, 2018 : 33). Inscrit encore dans la dialectique de la vie et de la mort, le nu artistique étend la quête du salut interculturel à l'acte primordial de la création divine souvent évoquée sous la formule «L'atelier argileux » (Daoud, 2018 : 22). Le corps pictural composite de « Marie-Houri » met en vis-à-vis deux visions du monde manifestement divergentes,

l'Au-delà et l'Ici-bas respectivement incarnés par chacune de ces deux figures

féminines. La fusion de celles-ci, dans un seul portrait artistique, signifie aussi concordance des deux imaginaires, celui de la mort (Au-delà) et de la vie (Ici-bas), un jumelage que résume le propos de « Marie Thérèse est peinte comme une houri, mais

Cette peinture consacre ainsi le rapprochement entre deux cultures d'orientations différentes, un dialogue interculturel atténuant par une telle démarche artistique la projection de l'imaginaire musulman dans la vie post-mortem 
, et la projection de l'imaginaire occidental dans la vie Ici-bas. L'allégorie du corps féminin n'est pas la seule manière, indirecte, de faire dialoguer les deux imaginaires évoqués. L'introduction decedialogue se fait également sans détour, en mettant côte à côte le religieux et l'art de la peinture, une symbiose interculturelle métonymiquement indiquée par «le verset » et « le pinceau » dans la citation suivante : « Cette "tradition

" me fascine. Elle mêle sans le savoir la peinture au verset, le coup de pinceau à la mort » (Daoud, 2018 : 154). L'interaction verset / peinture, pinceau / mort révèle, selon les termes de cette citation, l'inscription de l'interculturel dans l'acte créateur, divin, primordial.

Le mixage des cultures orientale et occidentale s'accentue par le biais de la calligraphie arabe décrite dans l'œuvre de Kamel Daoud comme une pratique biaisée du nu artistique. Une identité artistique, commune, confirmée par Picasso lui-même en qualifiant la calligraphie en question d' " Art érotique sublimé » (Daoud, 2018 : 162). Née dans la culture musulmane, la calligraphie se fait art qui ruse en permanence avec l'interdit religieux, une expression travestie de ce que cette culture encore rattachée au religieux empêcherait d'être. Cette souplesse culturelle est défendue par Fatima Mernissi, la sociologue marocaine spécialiste de la pensée islamique, en affirmant que « Contrairement à l'idée reçue, l'Islam possède une riche tradition de peintures profanes, et qu'il ne condamne les images figuratives que dans le domaine religieux » (Mernissi, 2001 : 21). Poussée à son expression extrême chez Kamel Daoud, puisque directement reliée au nu de Picasso, cette calligraphie prend la forme d'une expression alambiquée de la chair, que par ailleurs l'art du nu représente plus directement. La citation qui suit illustre ce cas de figure :

La calligraphie a lentement au fil des siècles pris les traits du nu désincarné et réincarné, la langueur, l'allongement, l'exposition de la toison et de l'érection, du sein et de la courbe, du pubis et du point. Elle se fait clandestinité publique, exposition aveuglante, ruse du corps dans le demi-mot (Daoud, 2018 : 163).

La définition de la calligraphie comme expression déformée du nu artistique atteste le point culminant d'harmonisation des civilisations occidentale et orientale, cette dernière demeure encore sous l'influence de l'Islam. La stratégie inter-artistique ainsi mise en œuvre à l'œuvre renforce significativement l'esthétique interculturelle, multiforme, dans les deux romans de Kamel Daoud.

En guise de conclusion, il nous semble avoir mis au jour la complexité de l'écriture de Zabor ou Les PsaumesetLe peintre dévorant la femme

de Kamel Daoud.Nous y avons décelé deux formes d'expressions, contradictoires, cellemilitante, satirique, qui rabaisse en permanence le sacré, et celle créatrice, qui transforme ce sacré en une symbiose interculturelle. Dans ce sens, le rapprochement des imaginaires oriental et occidental par le biais du dialogue inauguré entre les arts, les contes et les écritures imprègne le projet interculturel de Kamel Daoud. Ce projet interculturel, salvateur, ainsi investi, inscrit dans la durée la réflexion de Mouloud

Mammeri, celle relative au sort réservé - à l'ère contemporaine particulièrement marquée par l'uniformisation et l'hégémonisme - aux anciennes cultures. En effet, Mouloud Mammerise demande si : « Dans la culture barbare, que nous exécutons d'une giclée de canon dédaigneuse, n'y avait-il pas une formule de notre salut » (Mammeri, 1973 : 16). En définitive, ce débat culturel se poursuit avec encore plus de détermination dans les écrits récents d'Amine Zaoui, un autre écrivain algérien d'expression arabe et française. Ses deux essais intitulés : La boîte noire de l'islam. Le sacré et la discorde contemporaine(2018) et 
Dieu n'habite pas la Mecque. Pour une déconstruction du sacré

(2019) inscrivent résolument la thématique du sacré dans la dynamique interculturelle

en cours.

\section{BIBLIOGRAPHIE}

BARTHES, R., «L'ancienne rhétorique : aide-mémoire », inL'aventure sémiologique,

Paris, Seuil, 1985.

BENCHEIKH, J.,Les Mille et Une Nuits ou la parole prisonnière,Paris, Gallimard, 1988.

BRETON, A.,Manifeste du surréalisme,Paris, Gallimard, 1970.

DAOUD, K.,Le peintre dévorant la femme,Alger, Barzakh, 2018.

DAOUD, K.,Zabor ou Les Psaumes,Alger, Barzakh, 2017.

ELIADE, M.,Aspects du mythe,Paris, Gallimard, 1963.

GENETTE, G.,Palimpsestes. La littérature au second degré.Paris, Seuil, 1982.

JANKELEVITCH, V,L'ironie,Paris, Flammarion « Champs », 1964.

Le petit Robert de la langue française, Edition 2015.

MAMMERI, M.,La mort absurde des Aztèques,Paris, Librairie Académique Perrin, 1973.

MERNISSI, F,Le Harem et l'Occident,Paris, Albin Michel, 2001.

ZAOUI, A.,Allah n'habite pas la Mecque. Pour la déconstruction du sacré,Alger, Tafat, 2019.

ZAOUI, A.,La boîte noire de l'Islam. Le sacré et la discorde contemporaine,Alger, Tafat, 2018.

\section{NOTES}

1. Le hadith est « un recueil des actes et paroles du prophète Mohammed. Dans le courant sunnite les hadiths complètent le Coran ", Le petit Robert de la langue française, Edition 2015, p. 1209.

2. «La Robinsonnade est un genre littéraire, mais aussi cinématographique, qui tient son nom de Robinson Crusoé, roman de Daniel Defoe publié en 1719. La Robinsonnade est parfois considérée comme un sous-genre du roman d'aventures (ou du film d'aventure). Le terme a été créé et utilisé pour la première fois en 1731 Par l'écrivain allemand Johann Gottfried Schnabel », https:// fr.m.wikipedia.org/wiki/1 


\section{RÉSUMÉS}

L'écriture des romansZabor ou Les PsaumesetLe peintre dévorant la femme

de Kamel Daoud revêt un caractère équivoque, un projet créateur écartelé entre une expression militante amplement satirique et une orientation interculturelle esthétiquement travaillée. En apparence, cette écriture se présente sous la forme d'un discours satirique qui cible le sacré, soit en tenant un discours offensant, sarcastique, soit en se livrant à un jeu intertextuel marqué par le travestissement burlesque, la transposition thématique et la citation ironique. En filigrane, cette écriture est également une esthétique dans laquelle le sacré se transmue en un dialogue interculturel, qui vise à harmoniser les imaginaires oriental et occidental. Ce dialogue des cultures est sous-tendu par la fusion des arts du nu pictural et de la calligraphie arabe, de la robinsonnade et des Mille et Une Nuits, du genre romanesque et de l'ésotérisme de la mystique musulmane.

The writing of the novels Zabor or The Psalms and The Painter Devouring the Woman by Kamel Daoud takes on an equivocal character, a creative project torn between an amply satirical militant expression and an aesthetically crafted intercultural orientation. Apparently, this writing takes the form of a satirical discourse that targets the sacred, either by either by holding an offensive, sarcastic discourse, or by engaging in an intertextual game marked by the burlesque disguise, the thematic transposition and the ironic quote. Implicitly, this writing is also an aesthetic in which the sacred is transmuted into an intercultural dialogue which aims to harmonize the Eastern and Western imaginations. This dialogue of cultures is underpinned by the fusion of the arts of the pictorial nude and Arabic calligraphy, of the robinsonnade and One Thousand and One Nights, the novelistic genre and the esotericism of Muslim mysticism.

\section{INDEX}

Keywords : sacre, satire, creation, intercultural, Kamel Daoud

Mots-clés : sacré, satire, création, interculturel, Kamel Daoud

\section{AUTEUR}

\section{SMAIL MAHFOUF}

Université de Bejaia 